Research Article

Open Access

Special Issue Dedicated to Charles R. Johnson

Milica Anđelič*, Tamara Koledin, and Zoran Stanić

\title{
A note on the eigenvalue free intervals of some classes of signed threshold graphs
}

https://doi.org/10.1515/spma-2019-0014

Received September 2, 2019; accepted November 21, 2019

Abstract: We consider a particular class of signed threshold graphs and their eigenvalues. If $\dot{G}$ is such a threshold graph and $Q(\dot{G})$ is a quotient matrix that arises from the equitable partition of $\dot{G}$, then we use a sequence of elementary matrix operations to prove that the matrix $Q(\dot{G})-x I(x \in \mathbb{R})$ is row equivalent to a tridiagonal matrix whose determinant is, under certain conditions, of the constant sign. In this way we determine certain intervals in which $\dot{G}$ has no eigenvalues.

Keywords: signed graph; threshold graph; tridiagonal matrix; eigenvalue interval

MSC: 05C50, 05C22

Dedicated to Charles R. Jonhnon on the occasion of his 70th birthday.

\section{Introduction}

A signed graph $\dot{G}$ is a pair $(G, \sigma)$, where $G=(V, E)$ is an (unsigned) graph, and $\sigma: E \longrightarrow\{+1,-1\}$ is the signature or the sign function. The edge set of a signed graph is composed of subsets of positive and negative edges. Throughout the paper we interpret a graph as a signed graph with all the edges being positive.

The adjacency matrix $A_{\dot{G}}$ of $\dot{G}$ is obtained from the adjacency matrix of its underlying graph by reversing the sign of all 1's which correspond to negative edges. The eigenvalues of $\dot{G}$ are identified to be the eigenvalues of $A_{\dot{G}}$.

A threshold graph (also known as a nested split graph) is a $\left\{2 K_{2}, P_{4}, C_{4}\right\}$-free graph, i.e., it does not contain any of listed graphs as an induced subgraph. To explain the structure of threshold graphs, we partition their set of vertices into the $2 h$ cells $U_{1}, U_{2}, \ldots, U_{h}, V_{1}, V_{2}, \ldots, V_{h}$. Now, the subgraph induced by $\bigcup_{i=1}^{h} V_{i}$ (resp. $\bigcup_{i=1}^{h} U_{i}$ ) is complete (resp. totally disconnected), while all the vertices in $U_{i}$ are adjacent to all the vertices in $V_{j}$ precisely when $i \leq j$. The size of $U_{i}$ is denoted by $t_{i}$ and the size of $V_{j}$ is denoted by $s_{j}$. A schematic representation of a threshold graph is illustrated in Fig 1. For more details on these graphs (including a wide branch of applications) we refer the reader to $[10,11]$.

Observe that a threshold graph is connected whenever $s_{h} \geq 1$. Since every disconnected threshold graph is composed of a connected one and a set of isolated vertices, in this paper we restrict ourselves only to connected ones. We also assume that all the cells are non-empty.

In what follows we consider the class of signed threshold graphs with the following signature:

- the edges between different cells are always positive;

- the edges within each cell $V_{i}$ are either all positive or all negative.

`Corresponding Author: Milica Anđelić: Department of Mathematics, Kuwait University, Safat 13060, Kuwait,

E-mail: milica.andelic@ku.edu.kw

Tamara Koledin: Faculty of Electrical Engineering, University of Belgrade, Bulevar kralja Aleksandra 73, Belgrade, Serbia, E-mail: tamara@etf.rs

Zoran Stanić: Faculty of Mathematics, University of Belgrade, Studentski trg 16, Belgrade, Serbia, E-mail: zstanic@math.rs 


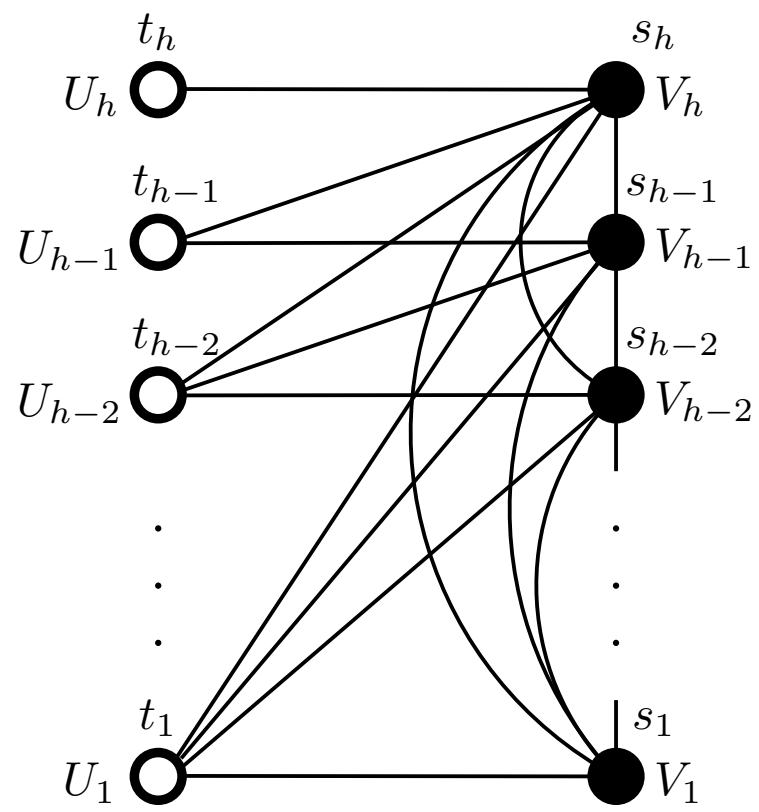

Figure 1: The structure of a threshold graph.

We denote this class by $\mathcal{T}$.

The problem of finding eigenvalue-free intervals has already been considered for threshold graphs with respect to the spectrum of the adjacency matrix in $[1,6,7]$. If we restrict ourselves to signed graphs, then there are no relevant references. This paper is prepared in the same spirit as [2], where the eigenvalue-free interval for threshold graphs (and chain graphs) with respect to distance spectrum were considered.

The paper is organized as follows. Two preliminary results are stated in the short Section 2. In Section 3 we consider the characteristic polynomial of the adjacency matrix of a signed threshold graphs defined as above. In Section 4 we deal with the interval $(-1,0)$, and we also consider the multiplicity of the eigenvalues 0 and -1 . In Section 5 we show that in certain intervals the characteristic polynomials of our signed threshold graphs have constant sign, which proves that these intervals are eigenvalue free. Finally, in Section 6 we give some concluding remarks on multiplicity of the eigenvalues.

\section{Background}

Let $M$ denote an $n \times n$ real symmetric matrix, and let its rows and columns be indexed by $S=\{1,2, \ldots, n\}$. Let further $S_{1} \sqcup S_{2} \sqcup \cdots \sqcup S_{k}$ be a partition of $S$, which determines a blocking of $M$ into the blocks $M_{i, j}$. If each block $M_{i, j}$ has a constant row sum, say $m_{i, j}$, the corresponding partition is called equitable, and the $k \times k$ matrix $Q=\left[m_{i, j}\right]$ is called the quotient matrix of $M$. The following result can be found in Section 2.3 of [3] (see also [4]).

Theorem 2.1. [3] Let $M$ be a real symmetric matrix with a quotient matrix $Q$. Then the characteristic polynomial of $Q$ divides the characteristic polynomial of $M$.

The next theorem gives sufficient conditions for a tridiagonal matrix (with positive diagonal entries) to have positive determinant. 
Theorem 2.2. [9] Let $M=\left[m_{i, j}\right]$ be an $n \times n$ real tridiagonal matrix with positive diagonal entries. If

$$
m_{i, i-1} m_{i-1, i}<\frac{1}{4} m_{i, i} m_{i-1, i-1} \frac{1}{\cos ^{2}\left(\frac{\pi}{n+1}\right)}, \quad \text { for } \quad 2 \leq i \leq n,
$$

then $\operatorname{det}(M)>0$.

\section{The characteristic polynomial of signed graphs in $\mathcal{T}$}

Let $u$ and $v$ be the vertices belonging to the same cell of a signed threshold graph $\dot{G} \in \mathcal{T}$. Consider the vector $\mathbf{x}$ defined in the following way: all its coordinates are zero except for those corresponding to $u$ and $v$ which are taken to be 1 and -1 . If $\mathbf{w}$ is the row of $A_{\dot{G}}$ corresponding to some vertex $w \notin\{u, v\}$, then $\mathbf{w} \cdot \mathbf{x}=0$ (where - stands for the standard inner product). Also, if $\mathbf{u}$ (resp. $\mathbf{v})$ is the row of $A_{\dot{G}}$ corresponding to the vertex $u$ (resp. $v$ ), then $\mathbf{u} \cdot \mathbf{x}=\mathbf{v} \cdot \mathbf{x}=0$, if $u, v \in U_{i}, \mathbf{u} \cdot \mathbf{x}=1, \mathbf{v} \cdot \mathbf{x}=-1$ if $u, v \in V_{i}$ with negative edges and $\mathbf{u} \cdot \mathbf{x}=-1$, $\mathbf{v} \cdot \mathbf{x}=1$ if $u, v \in V_{i}$ with positive edges. Thus, $\mathbf{x}$ is an eigenvector of the $A_{\dot{G}}$ associated to the eigenvalue 0,1 or -1 (depending on which cell selected vertices belong to). We record this in the following lemma.

Lemma 3.1. For $\dot{G} \in \mathcal{T}$, let $\{1,2, \ldots h\}=I \sqcup J$, such that for all $i \in I, V_{i}$ has negative edges, and for all $j \in J$ either the edges of $V_{j}$ are positive or $s_{j}=1$ (for short, $V_{j}$ has no negative edges). Then:

(i) 0 is an eigenvalue of $\dot{G}$ with multiplicity at least $\sum_{i=1}^{h}\left(t_{i}-1\right)$;

(ii) 1 is an eigenvalue of $\dot{G}$ with multiplicity at least $\sum_{i \in I}\left(s_{i}-1\right)$;

(iii) -1 is an eigenvalue of $\dot{G}$ with multiplicity at least $\sum_{j \in J}\left(s_{j}-1\right)$.

To consider the remaining eigenvalues, we first observe that the partition $U_{1} \cup V_{1} \cup U_{2} \cup V_{2} \cup \cdots \cup U_{h} \cup V_{h}$ of the vertex set of $\dot{G}$ induces an equitable partition of its adjacency matrix. The corresponding quotient matrix $Q(=Q(\dot{G}))$ is given by

$$
Q(\dot{G})=\left[\begin{array}{ccccccccc}
0 & s_{1} & 0 & s_{2} & 0 & \cdots & s_{h-1} & 0 & s_{h} \\
t_{1} & \varepsilon_{1}\left(s_{1}-1\right) & 0 & s_{2} & 0 & \cdots & s_{h-1} & 0 & s_{h} \\
0 & 0 & 0 & s_{2} & 0 & \cdots & s_{h-1} & 0 & s_{h} \\
t_{1} & s_{1} & t_{2} & \varepsilon_{2}\left(s_{2}-1\right) & 0 & \cdots & s_{h-1} & 0 & s_{h} \\
& & & & & & & & \\
\vdots & & & & & \ddots & & & \vdots \\
0 & 0 & 0 & 0 & 0 & \cdots & 0 & 0 & s_{h} \\
t_{1} & s_{1} & t_{2} & s_{2} & t_{3} & \cdots & s_{h-1} & t_{h} & \varepsilon_{h}\left(s_{h}-1\right)
\end{array}\right],
$$

where $\varepsilon_{k}=1$ if $k \in J$ and $\varepsilon_{k}=-1$ if $k \in I$. In other words,

$$
Q(\dot{G})_{2 i-1,2 j-1}=0, \quad Q(\dot{G})_{2 i-1,2 j}=\left\{\begin{array}{ll}
s_{j} & \text { if } i \leq j, \\
0 & \text { if } i>j,
\end{array} \quad Q(\dot{G})_{2 i, 2 j-1}=\left\{\begin{array}{cc}
t_{i} & \text { if } i \geq j, \\
0 & \text { if } i<j
\end{array}\right.\right.
$$

and

$$
Q(\dot{G})_{2 i, 2 j}= \begin{cases}s_{i}-1 & \text { if } i=j \text { and the edges of } V_{i} \text { are positive, } \\ 1-s_{i} & \text { if } i=j \text { and the edges of } V_{i} \text { are negative, } \\ s_{j} & \text { if } i \neq j .\end{cases}
$$

We can now define two types of eigenvalues of $A_{\dot{G}}$ according to their associated eigenvectors. According to Theorem 2.1 each one of $2 h$ eigenvalues of $Q(\dot{G})$ is also an eigenvalue of $A_{\dot{G}}$. These eigenvalues are of the first type: every eigenvector of $A_{\dot{G}}$ corresponding to an eigenvalue of its quotient matrix $Q(\dot{G})$ is constant on the parts of the partition, i.e. the coordinates corresponding to vertices belonging to the same cell are equal. With the procedure explained at the beginning of Section 2 we can define the $n-2 h$ linearly independent 
eigenvectors associated to the eigenvalues 0,1 or -1 of the second type, all of them being orthogonal to the eigenvectors corresponding to the eigenvalues of the first type (i.e. their coordinates sum up to zero on each part of the partition). From these observations and from Lemma 3.1 and Theorem 2.1 it now easily follows that the characteristic polynomial of $\dot{G}$ is given by

$$
\phi\left(A_{\dot{G}}, x\right)=x^{t-h}(x+1)^{\alpha}(x-1)^{\beta} \phi(Q(\dot{G}), x),
$$

where $t=\sum_{i=1}^{h} t_{i}, \alpha$ is the number of vertices in cells with negative edges and $\beta$ is the number vertices in cells with positive edges. Of course, for $s=\sum_{i=1}^{h} s_{i}, \alpha+\beta=s-h$.

We next apply a sequence of elementary row operations to simplify the computation of $\phi(Q(\dot{G}), x)=$ $\operatorname{det}\left(Q(\dot{G})-x I_{2 h}\right)$. We first perform:

- $R_{2 i-1} \leftarrow R_{2 i-1}-R_{2 i+1}$, for $1 \leq i \leq h-1$,

$-R_{2 h-1} \leftarrow R_{2 h-1}$,

$-R_{2} \leftarrow R_{2}$,

- $R_{2 i} \leftarrow R_{2 i}-R_{2 i-2}$, for $2 \leq i \leq h$

and we obtain

$$
\left[\begin{array}{cccccccc}
-x & s_{1} & x & 0 & 0 & \cdots & 0 & 0 \\
t_{1} & \varepsilon_{1} s_{1}-\varepsilon_{1}-x & 0 & s_{2} & 0 & \cdots & 0 & s_{h} \\
0 & 0 & -\chi & s_{2} & x & \cdots & 0 & 0 \\
0 & \left(1-\varepsilon_{1}\right) s_{1}+\varepsilon_{1}+\chi & t_{2} & \left(\varepsilon_{2}-1\right) s_{2}-\varepsilon_{2}-x & 0 & \cdots & 0 & 0 \\
& & & & & & & \vdots \\
\vdots & & & & & \ddots & & \vdots \\
0 & 0 & 0 & 0 & 0 & \cdots & -\chi & s_{h} \\
0 & 0 & 0 & 0 & 0 & \cdots & t_{h} & \left(\varepsilon_{h}-1\right) s_{h}-\varepsilon_{h}-x
\end{array}\right] .
$$

We proceed with:

- $R_{2 i-1} \leftrightarrow R_{2 i}$, for $1 \leq i \leq h$,

giving

$$
\left[\begin{array}{cccccccc}
t_{1} & \varepsilon_{1} s_{1}-\varepsilon_{1}-x & 0 & s_{2} & 0 & \cdots & 0 & s_{h} \\
-\chi & s_{1} & x & 0 & 0 & \cdots & 0 & 0 \\
0 & \left(1-\varepsilon_{1}\right) s_{1}+\varepsilon_{1}+x & t_{2} & \left(\varepsilon_{2}-1\right) s_{2}-\varepsilon_{2}-x & 0 & \cdots & 0 & 0 \\
0 & 0 & -\chi & s_{2} & x & \cdots & 0 & 0 \\
& & & & & & & \\
\vdots & & & & & \ddots & & \vdots \\
0 & 0 & 0 & 0 & 0 & \cdots & t_{h} & \left(\varepsilon_{h}-1\right) s_{h}-\varepsilon_{h}-x \\
0 & 0 & 0 & 0 & 0 & \cdots & -x & s_{h}
\end{array}\right] .
$$

Finally, by

- $R_{1} \leftarrow R_{1}-\sum_{i=1}^{h} R_{2 i}$, 
we obtain the tridiagonal matrix

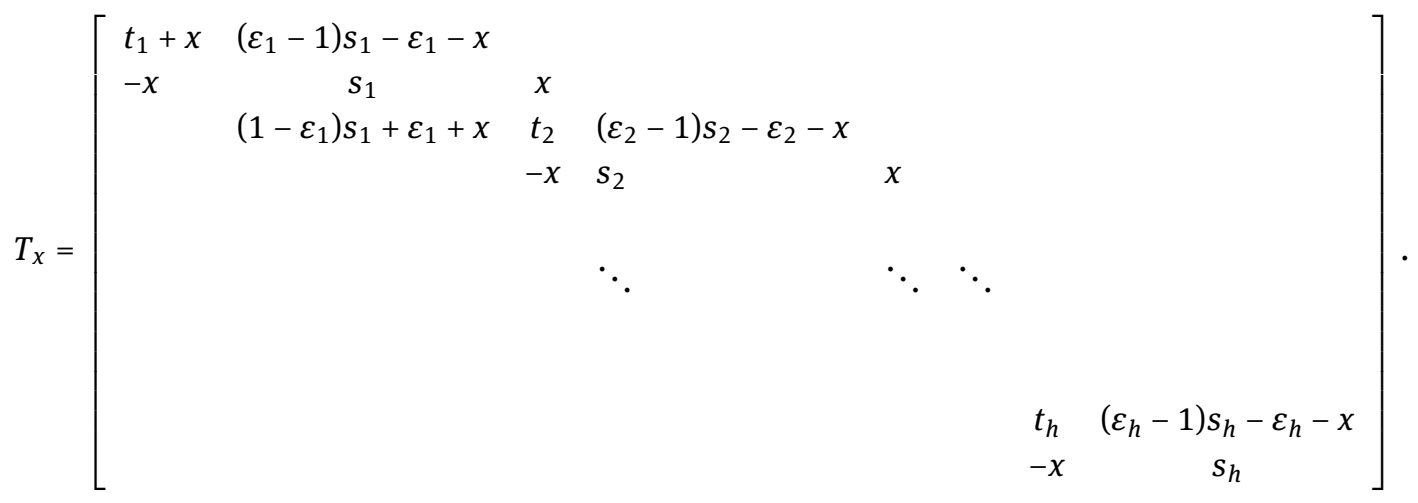

Now, by (3.1), we obtain

$$
\phi\left(A_{\dot{G}}, x\right)=(-1)^{h} x^{t-h}(x+1)^{\alpha}(x-1)^{\beta} \operatorname{det}\left(T_{x}\right) .
$$

\section{The interval $(-1,0)$ and multiplicity of 0 and -1}

We consider the sign of $\operatorname{det}\left(T_{X}\right)$. Denote further

$$
c_{h}=\frac{1}{4 \cos ^{2}\left(\frac{\pi}{2 h+1}\right)},
$$

and let $I$ and $J$ be as in Lemma 3.1. According to Theorem 2.2, we have $\operatorname{det}\left(T_{x}\right)>0$ whenever the following inequalities hold:

1. $x\left(x+2 s_{1}-1\right)<c_{h}\left(t_{1}+x\right) s_{1}$, if $V_{1}$ has negative edges and $x(x+1)<c_{h}\left(t_{1}+x\right) s_{1}$, otherwise,

2. $x\left(x+2 s_{i}-1\right)<c_{h} t_{i} s_{i}$, for $i \in I \backslash\{1\}$,

3. $x\left(x+2 s_{i}-1\right)<c_{h} t_{i+1} s_{i}$, for $i \in I \backslash\{1\}$,

4. $x(x+1)<c_{h} t_{j} s_{j}$, for $j \in J \backslash\{1\}$,

5. $x(x+1)<c_{h} t_{j+1} s_{j}$, for $j \in J \backslash\{1\}$,

6. $t_{1}+x>0$.

First, notice that, since $t_{1} \geq 1$, we have $t_{1}+x>0$, for $x \in(-1,0]$. Also, for $x<0$, we have $x\left(x+2 s_{i}-1\right) \leq x(x+1)$ (as $s_{i} \geq 1$ holds for $\left.1 \leq i \leq h\right)$. Thus, for $x \in(-1,0]$, all the left hand sides of the above inequalities are negative, while the right hand sides are positive; hence, $\operatorname{det}\left(T_{x}\right)>0$. This precisely means that $\phi(Q(\dot{G}), x)$ has no zeros in the interval $x \in(-1,0]$, and also that 0 is an eigenvalue of $\dot{G}$ with multiplicity exactly $\sum_{i=1}^{h}\left(t_{i}-1\right)$.

If, in addition, $t_{1}>1$, then $t_{1}-1>0$, and in a very similar way we deduce that $\operatorname{det}\left(T_{-1}\right)>0$ meaning that, in this case, -1 is not a zero of $\phi(Q(\dot{G}), x)$, i.e., it is an eigenvalue of $\dot{G}$ with multiplicity exactly $\alpha$.

In what follows, we consider the case $t_{1}=1 \mathrm{in}$ more details. Suppose first that $V_{1}$ has no negative edges, and take into account the following submatrices of $T_{\chi}$ :

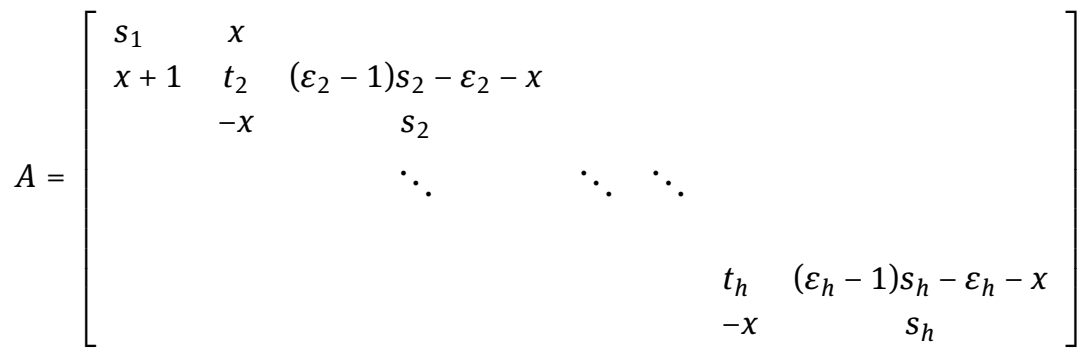


of the size $2 h-1$ and

$$
B=\left[\begin{array}{ccccccc}
t_{2} & \left(\varepsilon_{2}-1\right) s_{2}-\varepsilon_{2}-x & & & & & \\
-x & s_{2} & x & & & & \\
& \left(1-\varepsilon_{2}\right) s_{2}+\varepsilon_{2}+x & t_{3} & & & & \\
& & \ddots & \ddots & \ddots & & \\
& & & & & & \\
& & & & & t_{h} & \left(\varepsilon_{h}-1\right) s_{h}-\varepsilon_{h}-x \\
& & & & & -x & s_{h}
\end{array}\right]
$$

of the size $2 h-2$.

Using the Laplacian expansion of $\operatorname{det}\left(T_{x}\right)$ along the first row, we obtain $\operatorname{det}\left(T_{x}\right)=(1+x)(\operatorname{det}(A)-$ $x \operatorname{det}(B))$. It is clear that, in this case, $\operatorname{det}\left(T_{-1}\right)=0$, and so -1 is a zero of $\phi(Q(\dot{G}), x)$. It is also clear that both matrices $A$ and $B$ have positive diagonal, and that, for $x=-1$, we have $x\left(x+2 s_{i}-1\right) \leq x(x+1)=0<c_{h} t_{i} s_{i}$ and $x\left(x+2 s_{i}-1\right) \leq x(x+1)=0<c_{h} t_{i} s_{i-1}$, for all $2 \leq i \leq h$. According to Theorem 2.2, for $x=-1$, $\operatorname{det}(A)-x \operatorname{det}(B)>0$, so, -1 is a zero of $\phi(Q(\dot{G}), x)$ with multiplicity one. Thus, by (3.2), -1 is an eigenvalue of $\dot{G}$ with multiplicity exactly $\alpha+1$.

Suppose now that, for $t_{1}=1, V_{1}$ has negative edges (this in particular implies that $s_{1}>1$ ). We have $\operatorname{det}\left(T_{x}\right)=(1+x) \operatorname{det}\left(A^{\prime}\right)-x\left(2 s_{1}+x-1\right) \operatorname{det}(B)$, where $A^{\prime}$ differs from $A$ only in the (2,1)-entry, which is now $2 s_{1}+x-1$. Again, $B$ has a positive diagonal, and for $x=-1$, we have $x\left(x+2 s_{i}-1\right)<x(x+1)=0<c_{h} t_{i} s_{i}$ and $x\left(x+2 s_{i}-1\right)<x(x+1)=0<c_{h} t_{i} s_{i-1}$, for all $2 \leq i \leq h$. Thus, according to Theorem 2.2, we have $\operatorname{det}\left(T_{-1}\right)>0$, which means that -1 is not a zero of $\phi(Q(\dot{G}), x)$, and -1 is an eigenvalue of $\dot{G}$ with multiplicity exactly $\alpha$.

Gathering the above results, we arrive at the following.

Theorem 4.1. A signed graph $\dot{G} \in \mathcal{T}$ has no eigenvalues in $(-1,0)$. If for any $i \in\{2, \ldots h\}, V_{i}$ has no negative edges, then:

(i) 0 is an eigenvalue of $\dot{G}$ with multiplicity $\sum_{i=1}^{h}\left(t_{i}-1\right)$;

(ii) if either $t_{1}>1$ or $t_{1}=1$ and $V_{1}$ has negative edges, then -1 is an eigenvalue of $\dot{G}$ with multiplicity $\sum_{i \in I}\left(s_{i}-\right.$ 1);

(iii) if $t_{1}=1$ and $V_{1}$ has no negative edges, then -1 is an eigenvalue of $\dot{G}$ with multiplicity $1+\sum_{i \in I}\left(s_{i}-1\right)$.

Remark 4.2. If the signed threshold graph $\dot{G}$ has no negative edges, clearly $\phi\left(A_{\dot{G}}, x\right)=\phi\left(A_{G}, x\right)$. In this case, Theorem 4.1 recovers the corresponding results for unsigned graphs obtained by different methods in $[6,8]$.

\section{Eigenvalue free intervals}

We determine eigenvalue free intervals under the additional assumption that $t_{1}>1$ or $t_{1}=1$ and $V_{1}$ has no negative edges. First, we consider the interval $(-\infty, 0)$.

Suppose that $t_{1}=1$ and that $V_{1}$ has no negative edges. Recall that, in this case, there must be $\operatorname{det}\left(T_{-1}\right)=$ 0 , and recall the submatrices $A$ and $B$ of $T_{x}$ given by (4.2) and (4.3). We have $\operatorname{det}\left(T_{x}\right)=(1+x)(\operatorname{det}(A)-\chi \operatorname{det}(B))$, so if $x<-1$ and if $\operatorname{det}(A)$ and $\operatorname{det}(B)$ are positive, then it follows that $\operatorname{det}\left(T_{x}\right)$ is negative.

Consider now the positivity of $\operatorname{det}(A)$ and $\operatorname{det}(B)$. If the parameter $c_{h}$ is defined by (4.1) and $I \sqcup J$ represents the partition of $\{1,2, \ldots, h\}$ described in Lemma 3.1, then by Theorem 2.2, we conclude that both determinants are positive whenever the conditions (2)-(5) described in Section 4 hold.

Denote further

$$
a_{h}=\min _{2 \leq i \leq h-1}\left\{s_{1} t_{2}, s_{i} t_{i+1}, s_{i} t_{i}, s_{h} t_{h}\right\} .
$$

Since for $x<0$, we have $x\left(x+2 s_{i}-1\right)<x(x+1)$, it follows that both determinants are positive whenever $x \in\left(\frac{-1-\sqrt{1+4 c_{h} a_{h}}}{2},-1\right)$, which implies $\operatorname{det}\left(T_{x}\right) \neq 0$. In other words, $\dot{G}$ has no eigenvalues in that interval. 
Similarly, for $t_{1}>1$, we immediately get $\operatorname{det}\left(T_{-1}\right)>0$, and by Theorem 2.2, $\operatorname{det}\left(T_{X}\right)>0$ holds if the conditions (1)-(6) described in Section 4 hold.

Now, for $x<0$, we have $x\left(x+2 s_{i}-1\right)<x(x+1)$, and so $\operatorname{det}\left(T_{x}\right)>0$ whenever $x \in I_{1} \cap I_{2}$, where

$$
I_{1}=\left(\frac{-1-\sqrt{1+4 c_{h} a_{h}}}{2},-1\right), I_{2}=\left(-t_{1}, 0\right) \text {. }
$$

These considerations lead to the following theorem.

Theorem 5.1. For a signed graph $\dot{G} \in \mathcal{T}$, we have:

(i) if $t_{1}=1$ and $V_{1}$ has no negative edges, then $\dot{G}$ has no eigenvalues in $\left(\frac{-1-\sqrt{1+4 c_{h} a_{h}}}{2},-1\right)$;

(ii) if $t_{1}>1$, then $\dot{G}$ has no eigenvalues in $(-\mathcal{S},-1)$,

where the parameters $c_{h}$ and $a_{h}$ are defined by (4.1) and (5.1), respectively, and $\mathcal{S}=\min \left\{\frac{1+\sqrt{1+4 c_{h} a_{h}}}{2}, t_{1}\right\}$.

Since $4 c_{h} a_{h}>1$ and $\frac{-1-\sqrt{2}}{2}>-t_{1}$ (for $t_{1}>1$ ), we have a direct consequence.

Corollary 5.2. A signed graph $\dot{G} \in \mathcal{T}$ has no eigenvalues in $\left(\frac{-1-\sqrt{2}}{2},-1\right)$.

We proceed with the assumption that $x>0$. Here, $t_{1}+x>0$ and $T_{x}$ has a positive diagonal. By Theorem 2.2, we have $\operatorname{det}\left(T_{x}\right)>0$ whenever the conditions (1)-(5) described in Section 4 hold.

Observe that, for $x>0, x(x+1)<x\left(x+2 s_{i}-1\right)$ holds, and denote

$$
\begin{aligned}
\mathcal{M}= & \min _{2 \leq i \leq h-1}\left\{\frac{s_{1} c_{h}-2 s_{1}+1+\sqrt{\left(2 s_{1}-1-s_{1} c_{h}\right)^{2}+4 c_{h} t_{1} s_{1}}}{2},\right. \\
& \frac{1-2 s_{i}+\sqrt{\left(2 s_{i}-1\right)^{2}+4 c_{h} t_{i} s_{i}}}{2}, \frac{1-2 s_{i}+\sqrt{\left(2 s_{i}-1\right)^{2}+4 c_{h} t_{i+1} s_{i}}}{2}, \\
& \left.\frac{1-2 s_{h}+\sqrt{\left(2 s_{h}-1\right)^{2}+4 c_{h} t_{h} s_{h}}}{2}\right\} .
\end{aligned}
$$

It follows that $\operatorname{det}\left(T_{\chi}\right)>0$ holds whenever $x \in(0, \mathcal{M})$, which leads to the following theorem.

Theorem 5.3. A signed graph $\dot{G} \in \mathcal{T}$ has no eigenvalues in $(0, \mathcal{M})$, where the parameter $\mathcal{M}$ is defined in (5.2).

A similar reasoning leads to the conclusion that if $G$ is a threshold graph, then $\operatorname{det}\left(T_{x}\right)>0$ holds whenever the following inequalities hold: $x(x+1)<c_{h}\left(t_{1}+x\right) s_{1}, x(x+1)<c_{h} t_{j} s_{j}$ and $x(x+1)<c_{h} t_{j+1} s_{j}$. If $x>0$, then we have $c_{h} t_{1} s_{1}<c_{h}\left(t_{1}+x\right) s_{1}$, which implies the following.

Theorem 5.4. A threshold graph $G$ has no eigenvalues in $\left(0, \frac{-1+\sqrt{1+4 c_{h} a_{h}}}{2}\right)$, where the parameters $c_{h}$ and $a_{h}$ are defined by (4.1) and (5.1), respectively.

Again, the inequality $4 c_{h} a_{h}>1$ gives an immediate consequence.

Corollary 5.5. A threshold graph $G$ has no eigenvalues in $\left(0, \frac{-1+\sqrt{2}}{2}\right)$.

Remark 5.6. Corollary 5.2 covers a particular case of threshold graphs. In conjunction with Corollary 5.5, it leads to the conclusion that, apart from the eigenvalues -1 and 0 , the interval $\left(\frac{-1-\sqrt{2}}{2}, \frac{-1+\sqrt{2}}{2}\right)$ does not contain an eigenvalue of any threshold graph - a result obtained in [6].

\section{Some remarks on multiplicity of eigenvalues}

We conclude with the following observation. It is known from [8] that, in a threshold graph, all the eigenvalues distinct from 0 and -1 are simple. This is not true for signed threshold graphs. An example is a signed 
threshold graph of $\mathcal{T}$ determined by the parameters $h=3, s_{1}=2, s_{2}=8, s_{3}=1, t_{1}=3, t_{2}=1, t_{3}=9$, and in which, apart from those of $V_{1}$, all the edges are positive. Its eigenvalues (distinct from 0 and -1 ) are: $12.9050,2.3678,0.4248,-3.6977$ and -3 with multiplicity 2 .

On the other hand, we can easily see that a signed graph of $\mathcal{T}$ cannot have an eigenvalue (distinct from 0 and -1 ) with multiplicity greater than 2 . Indeed, considering the eigenvalue $\lambda \notin\{-1,0\}$, we $\operatorname{get} \operatorname{det}\left(T_{\lambda}\right)=0$. Now, if we delete the last two columns and the first and the last row from (the triagonal matrix) $T_{\lambda}$ (see the end of Section 3), then we obtain a diagonal matrix with non-zero diagonal elements, so the rank of $T_{\lambda}$ is at least $2 h-2$. Consequently, the multiplicity of $\lambda$ is at most 2 .

Acknowledgement The authors would like to thank the anonymous referees for careful reading and helpful comments and suggestions that lead to the improvement of the original manuscript.

The work of the second author is supported by Serbian Ministry of Education, Science and Technological Development, Project 174033. The work of the third author is supported by Serbian Ministry of Education, Science and Technological Development, Projects 174012 and 174033.

\section{References}

[1] C.O. Aguilar, J. Lee, E. Piato, B.J. Schweitzer, Spectral characterizations of anti-regular graphs, Linear Algebra Appl., 557 (2018), 84-104.

[2] A. Alazemi, M. Anđelić, T. Koledin, Z. K. Stanić, Eigenvalue-free intervals of distance matrices of threshold and chain graphs, Linear Multilinear Algebra, submitted.

[3] A.E. Brouwer, W.H. Haemers, Spectra of graphs, Springer, 2011.

[4] M. Anđelić, C. M. da Fonseca, Sufficient conditions for positive definiteness of tridiagonal matrices revisited, Positivity, 15 (2011), 155-159.

[5] D. Cvetković, P. Rowlinson, S.K. Simić, An Introduction to the Theory of Graph Spectra, Cambridge University Press, Cambridge, 2010.

[6] E. Ghorbani, Eigenvalue-free interval for threshold graphs, Linear Algebra Appl., 583 (2019), 300-305.

[7] D.P. Jacobs, V. Trevisan, and F. Tura, Eigenvalue location in threshold graphs, Linear Algebra Appl., 439 (2013), $2762-2773$.

[8] D.P. Jacobs, V. Trevisan, F. Tura, Eigenvalues and energy in threshold graphs, Linear Algebra Appl., 465 (2015), $412-425$.

[9] C.R. Johnson, M. Neumann, M.J. Tsatsomeros, Conditions for the positivity of determinants, Linear Multilinear Algebra, 40 (1996), 241-248.

[10] V.R. Mahadev, U.N. Peled, Threshold Graphs and Related Topics, North-Holland, Amsterdam, 1995.

[11] Z. Stanić, Inequalities for Graph Eigenvalues, Cambridge University Press, Cambridge, 2015. 